\title{
Quantitative Monitoring of Osseointegrated Implant Stability Using Vibration Analysis
}

\author{
Shouxun Lu ${ }^{1, a,{ }^{*}}$, Benjamin Steven Vien ${ }^{1, b}$, Matthias Russ ${ }^{2,3, c}$, Mark Fitzgerald ${ }^{2,3, d}$ \\ and Wing Kong Chiu ${ }^{1, e}$
}

\author{
${ }^{1}$ Department of Mechanical \& Aerospace Engineering, Monash University, Wellington Rd, \\ Clayton Vic 3800, Australia \\ ${ }^{2}$ The Alfred Hospital, 55 Commercial Road, Melbourne Vic 3004, Australia \\ ${ }^{3}$ National Trauma Research Institute, 89 Commercial Road, Melbourne Vic 3004, Australia \\ ashouxun.lu@monash.edu, bben.vien@monash.edu, cM.Russ@alfred.org.au, \\ dM.Fitzgerald@alfred.org.au, ewing.kong.chiu@monash.edu
}

\begin{abstract}
Keywords: Osseointegrated Implant, Vibrational Response, Vibration Analysis, Finite Element Modelling
\end{abstract}

\begin{abstract}
Reliable and quantitative assessments for the stability of the osseointegrated prostheses are desirable and advantageous in ensuring the success of the installation and longterm performance. However, the common evaluation techniques are qualitative, where their accuracy of which relies on the surgeon's experience. This computational study investigates the potential of using vibrational response to evaluate the stability of the osseointegrated implant using finite element simulation. This paper mainly focuses on the resonance frequency shift and mode shape changes associated with the degree of osseointegration which is simulated by varying bone-implant interface Young's modulus. The resonance frequency of the specific torsional modes increases $211 \%$ and $155 \%$ for low-frequency ( 0 to $1800 \mathrm{~Hz}$ ) and high-frequency $(1800$ to $5000 \mathrm{~Hz})$ ranges respectively, as the simulated osseointegration process. Moreover, the torsional mode change from the implant to the femur-implant system is clearly evidenced. The findings highlight the potential application of vibration analysis on the assessment of implant stability.
\end{abstract}

\section{Introduction}

Osseointegrated implant, of which intramedullary part inserting into the skeletal system directly, provides joint mobility and control of the prosthesis with the sensory feedback from the ground [1-3]. This surgical treatment is developed based on a biological phenomenon, which is known as "osseointegration". Osseointegration is a highly complex and dynamic process, which constructs a direct mechanical connection between bone and biocompatible material gradually [4-6]. This functional structure allows the load transmission through the bone-implant interface. The common material of the osseointegrated implant is a titanium alloy due to its great biocompatibility and high resistance to the repeated load and corrosion [3, 4, 7]. Even though the osseointegrated implant has been described as the preferred surgical treatment for the amputee, the success of the osseointegrated requires relative strict prerequisites such as sufficient primary (mechanical) stability to promote bone regeneration and remodeling [1, 8, 9]. Otherwise, the formation of fibrous tissue at the bone-implant interface will hinder the initial osseointegration $[3,10,11]$. After the accomplishment of primary stability, the osseointegration starts with bone tissue formation at the bone-implant interface. This mechanical structure requires adequate time for rehabilitation to be capable of the full weight of patient $[2,3,12]$. The integrity of this 
structure is also known as secondary stability and therefore, the secondary stability is highly related to the state of osseointegration.

The conventional methods used to assess the implant stability such as clinical X-ray, pull-out test and magnetic resonance imaging (MRI) are invasive and subjective where their accuracy is highly dependent on the surgeon's experience [1, 13-16]. Recently, there is a significant research interest in using non-invasive approaches to monitor implant stability. Vibration analysis is a non-destructive structural health monitoring technique, which was firstly used in evaluating bone fracture healing [17-19] and monitoring the total hip arthroplasty loosening [20-23]. The methodology of using the dynamic response of the bone-implant system to assess the stiffness at the bone-implant interface is widely recognized $[1,21,24]$. Recent researches have also shown that the stiffness change at the bone-implant interface was related to the state of osseointegration $[20,24,25]$. Shao. et. al.[1] conducted an experiment on an amputee during his rehabilitation process and their results illustrated a gradually increase in the resonance frequency until the implant is capable of full weight load bearing. Moreover, a previous experimental study on rabbit tibias, implants were installed into different size predrilled cavities to simulate secure-fit and loose-fit connection [26]. Their result demonstrated that the bone-implant system with the loosefit connection had lower initial resonance frequency compared to those with the secure-fit condition. In addition, the study conducted by Carins et. al.[14, 27] exhibited higher resonance frequencies and that the associated mode shapes are more sensitive to the stiffness change at the bone-implant interface. Similar findings were also reported in [21, 22, 25, 28, 29].

The objective of this paper is to investigate the vibrational response on monitoring the degree of osseointegration at the bone-implant interface via finite element simulation. This vibrationalbased approach is based on the detection of the shift in resonance frequency and mode shape change to assess the level of osseointegration.

\section{Methodology}

In the computational analysis, the bone model used in this project was based on an artificial femur model scanned by a structured light 3D scanner. The femur model consists of two parts: the cortical shell and spongy bone (see Fig. 1a). The material of femur-implant model was assumed to be homogeneous and isotropic [30, 31]. The material properties of the femur and implant system [32-34] are described in Table 1. The novel osseointegrated endoprosthesis implant model was developed based on the design concept proposed by Russ et al.[35]. As shown in Figure 1b, the novel implant model consists of two parts: Extramedullary (EM) strut and Intramedullary (IM) stem. The implant model was generated by using the Shell function in the SolidWorks. The contour of the EM strut and IM stem have certain tolerance with the outer surface of the cortical shell and medullary cavity for the application of femur-implant interface layer.

Table 1: Material properties of the femur and implant system.

\begin{tabular}{|c|c|c|}
\hline Material & Young's modulus[MPa] & Poisson's Ratio \\
\hline Cortical shell & 17600 & 0.3 \\
\hline Spongy bone & 13000 & 0.36 \\
\hline Titanium alloy & 113800 & 0.342 \\
\hline
\end{tabular}

A thin layer, which used to simulate the osseointegration, was applied to the femur-implant interface, as illustrated in Fig 2. The layer thickness at EM strut and IM stem surfaces were set to 
$0.985 \mathrm{~mm}$ and $0.5 \mathrm{~mm}$, respectively. The surfaces of the femur-implant interface layer were bonded with both implant and femur model.

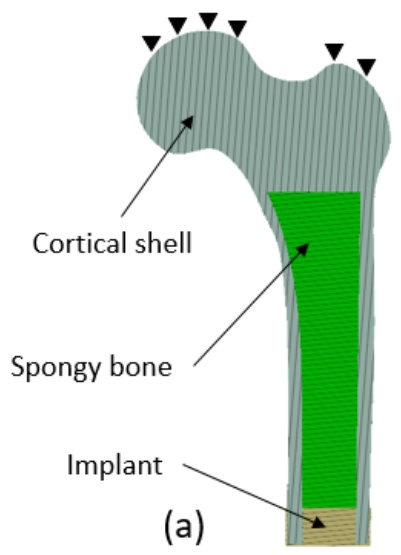

(b)

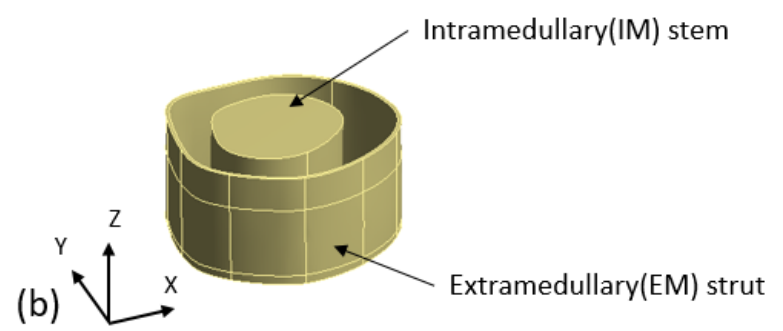

Fig. 1: (a) Cross-section of the computational femur model and (b) Implant.

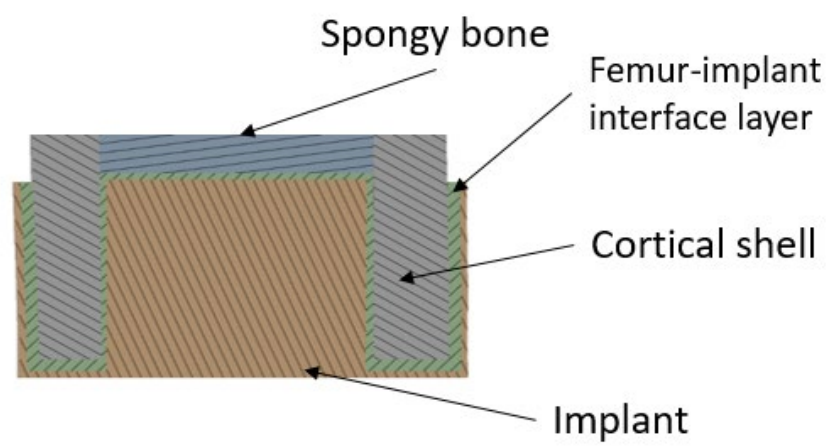

Fig. 2: Cross-section of the femur-implant interface.

ANSYS 19.0 Modal analysis was performed to determine the dynamic response of the femurimplant model. The femur model was fixed at the proximal end to simulate constraints from muscle and pelvis, as illustrated in Fig. 1a. The type of element was set to "Quadratic Tetrahedrons" to avoid stress singularity at sharp corners. The global mesh size was set to $5 \mathrm{~mm}$, which was optimized by a mesh convergence test with 5\% convergence error for the maximum von Misses stress on the femur. In addition, the mesh around the interface was further refined with a localized body sizing of $1.5 \mathrm{~mm}$. The modal analysis mainly focused on the frequency range of 0 to $5000 \mathrm{~Hz}$ as mentioned in $[14,27]$.

This computational analysis aims to investigate the resonance frequency shift and mode shape change under various conditions of primary and secondary stability. Hence, as illustrated in Table 2, Young's modulus $(E)$ of femur-implant interface layer was varied from $0.001 \%$ to $100 \%$ that of the cortical shell $\left(E_{c}\right)$ were investigated to simulate the process from $0 \%$ osseointegration to fully osseointegrated implant as mentioned in [36]. The method of employing the variation in $E$, is similar to the application of adhesive at the femur-implant interface to simulate the process of osseointegration in several experiments mentioned in [17, 37-39]. 
Table 2: Young's modulus of the layer (E) relative to that of cortical shell.

\begin{tabular}{|c|c|c|c|c|c|c|c|c|c|c|c|}
\hline Percentage[\%Ec] & 0.001 & 0.0025 & 0.005 & 0.0075 & 0.01 & 0.05 & 0.1 & 0.5 & 1 & 10 & 100 \\
\hline $\boldsymbol{E}[\mathbf{M P a}]$ & 0.176 & 0.44 & 0.88 & 1.32 & 1.76 & 8.8 & 17.6 & 88 & 176 & 1760 & 17600 \\
\hline
\end{tabular}

\section{Result}

The deformation in y-axis direction along z-axis was shown in Fig. 3. This deformation shows the presence of the torsional mode. The results demonstrated in Fig 3a and 3c, where the implant is not osseoinegrated with the femur, shows only the implant responding (TI). When the implant is fully osseointegrated, the torsion response of the entire femur-implant system (denoted as TS) is recorded (see Fig $3 b$ and $3 d$ ).
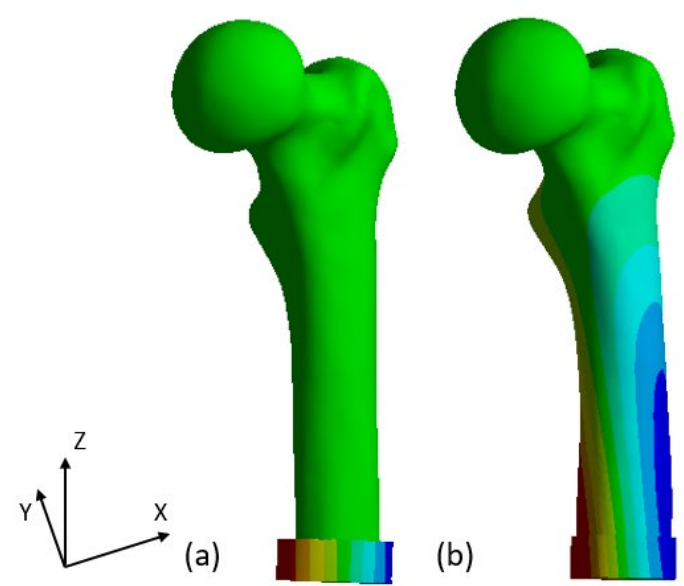

(c)
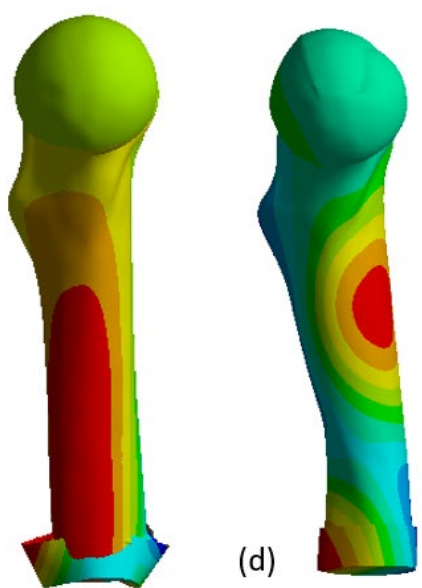

Fig. 3: Low-frequency torsional mode of: (a) implant (TI) with 0.001\%Ec; (b) femur-implant system (TS) with 100\%Ec and high-frequency: (c) TI with $0.0025 \%$ Ec; (d) TS with $100 \%$ Ec.

Figure 4 demonstrated the variation of the resonance frequency with the simulated osseointegration $(S O)$ process in both low-frequency $(0$ to $1800 \mathrm{~Hz})$ and high-frequency (1800 to $5000 \mathrm{~Hz}$ ) ranges. The percentage on the plot indicated that the resonance frequency gradually increased relative to the first torsional frequency, along the $S O$ process.

In the early stage of $S O$ process, the change of resonance frequency could be easily identified in both frequency ranges. The resonance frequency of the specific torsional modes increases $211 \%$ and $155 \%$ for low- and high-frequency ranges respectively, as the $S O$ process. As illustrated in Fig. 3a, there is a significant increase in the resonance frequency of $T I$ from $568.45 \mathrm{~Hz}$ to $1433.2 \mathrm{~Hz}$, as $E$ increased to $0.01 \% E_{c}$. Similar smooth variation in resonance frequency was identified in the high-frequency range. This result was in accordance with $[1,22$, 28], suggesting that the frequency shift of the system increases along the mode shape complexity increasing. The change of frequency indicated a clear correlation between the $E$ and resonance frequency in the early stage of $S O$ and the result agrees with the findings in Cairns et. al. study [14]. 


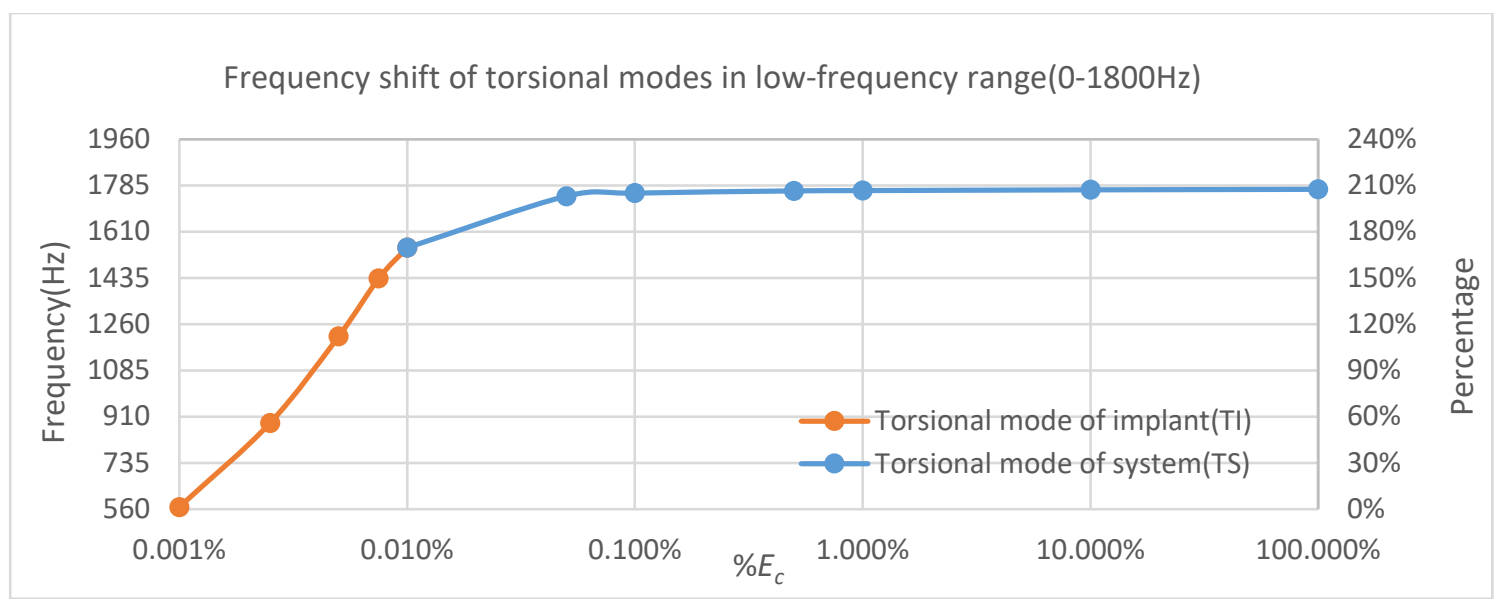

(a)

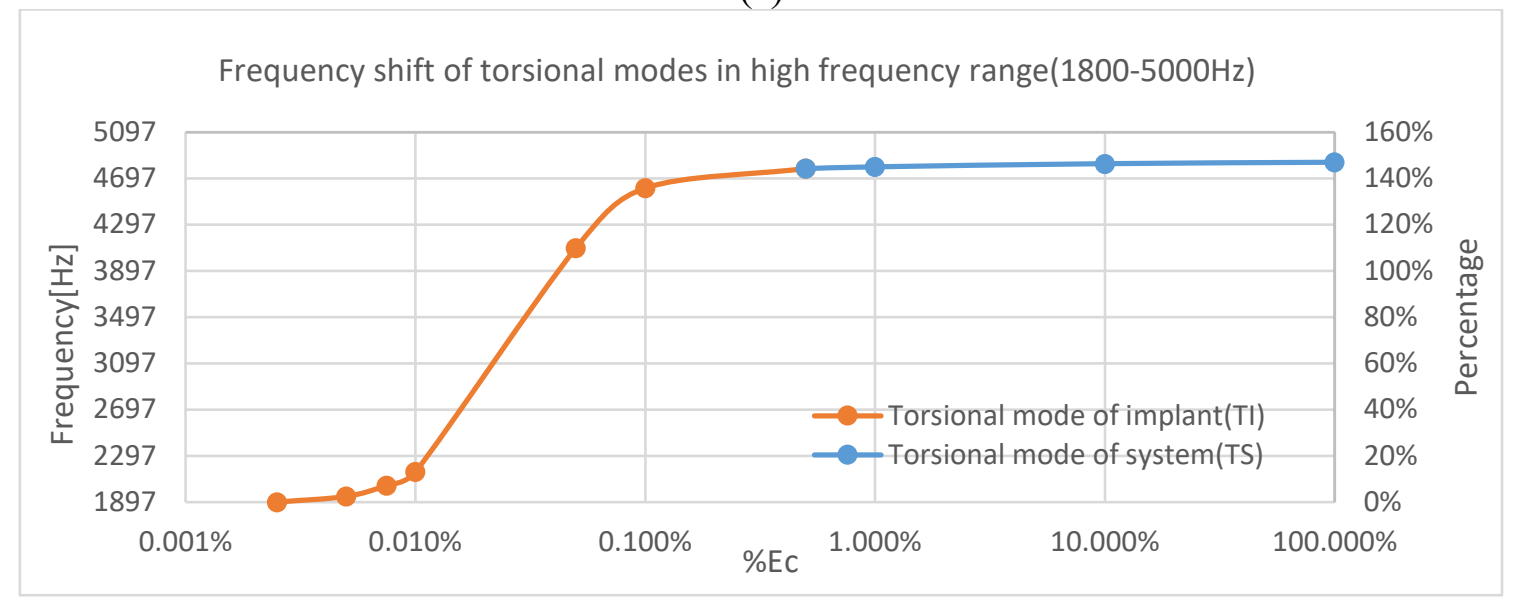

(b)

Fig. 4: Frequency shift of torsional modes in frequency range: (a) 0-1800Hz; (b) 1800-5000Hz.

Moreover, the mode located at $568.45 \mathrm{~Hz}$ was observed to initially vibrate as implant torsional mode $T I$ and then migrated to system torsional mode $T S$ at $1770.6 \mathrm{~Hz}$, with the process of $S O$. The transformation, from the significant vibration at implant to torsional mode of the femurimplant system, was apparent in high-frequency range as well. However, beyond $1 \% E_{c}$, the frequency shift caused by the change of $E$ was negligible for both low- and high-frequency ranges after the mode shape change.

Even though the sensitivity of this method was constrained in the relative small $E$ value, this variation in frequency and mode shape were significant, suggesting that monitoring the resonance frequency and mode shape change could aid in early detection of insufficient stability, thereby decreases the likelihood of failure in osseointegration.

\section{Conclusion}

This paper reveals the preliminary concept that monitoring the primary and secondary stability of the femur treated with novel implant design could be evaluated with the dynamic response of vibration. The finite element investigation has demonstrated that the resonance frequency increased gradually along the $S O$ process. The result has also demonstrated the significant shift in resonance frequency, $211 \%$ and $155 \%$ for low- and high-frequency ranges respectively, indicating the potential of vibration analysis on the quantitative evaluation of the implant 
stability at the early stage of osseointegration. Furthermore, the identification of mode shape change enhanced the evaluation of stimulated osseointegration process. Future work is currently in progress to investigate this vibrational analysis method on various length of residual femur.

\section{Funding}

This research is funded by US Navy Office of Naval Research (N62909-19-1-2051). The financial supported provided by the Office of Naval Research is gratefully acknowledged.

\section{Conflict of Interest}

The author declares that there is no conflict of interest regarding the publication of this paper.

\section{Ethical approval}

This article does not contain any studies with human participants or animals performed by any of the authors.

\section{References}

[1] Shao, F., et al., Natural frequency analysis of osseointegration for trans-femoral implant. Ann Biomed Eng, 2007. 35(5): p. 817-24. https://doi.org/10.1007/s10439-007-9276-z

[2] Hagberg, K., et al., Osseoperception and osseointegrated prosthetic limbs, in Psychoprosthetics. 2008, Springer. p. 131-140. https://doi.org/10.1007/978-1-84628-980-4_10

[3] Ward, D. and K. Robinson, Osseointegration for the skeletal fixation of limb prostheses in amputations at the trans-femoral level. The osseointegration book: From calvarium to calcaneus. Berlin (Germany): Quintessence, 2005: p. 463-76.

[4] Isaacson, B.M. and S. Jeyapalina, Osseointegration: a review of the fundamentals for assuring cementless skeletal fixation. Orthopedic Research and reviews, 2014. 6: p. 55-65. https://doi.org/10.2147/ORR.S59274

[5] Brånemark, R., et al., Osseointegration in skeletal reconstruction and rehabilitation: a review. Journal of rehabilitation research and development, 2001. 38(2): p. 175.

[6] Morelli, F., et al., Influence of bone marrow on osseointegration in long bones: an experimental study in sheep. Clinical oral implants research, 2015. 26(3): p. 300-306. https://doi.org/10.1111/clr.12487

[7] Agarwal, R. and A.J. García, Biomaterial strategies for engineering implants for enhanced osseointegration and bone repair. Advanced Drug Delivery Reviews, 2015. 94. https://doi.org/10.1016/j.addr.2015.03.013

[8] Lu, S., et al., Non-radiative healing assessment techniques for fractured long bones and osseointegrated implant. Biomedical engineering letters, 2020. 10(1): p. 63-81. https://doi.org/10.1007/s13534-019-00120-0

[9] Vien, B.S., et al., A stress wave-based health monitoring concept on a novel osseointegrated endoprosthesis design. 2018.

[10] Østbyhaug, P.O., et al., Primary stability of custom and anatomical uncemented femoral stems: A method for three-dimensional in vitro measurement of implant stability. Clinical Biomechanics, 2010. 25(4): p. 318-324. https://doi.org/10.1016/j.clinbiomech.2009.12.012 [11] Lioubavina-Hack, N., N.P. Lang, and T. Karring, Significance of primary stability for osseointegration of dental implants. Clinical Oral Implants Research, 2006. 17(3): p. 244-250. https://doi.org/10.1111/j.1600-0501.2005.01201.x 
[12] Thesleff, A., B. Håkansson, and M. Ortiz-Catalan, Biomechanical Characterisation of Boneanchored Implant Systems for Amputation Limb Prostheses: A Systematic Review. Annals of Biomedical Engineering, 2018. 46(3): p. 377-391. https://doi.org/10.1007/s10439-017-1976-4 [13] Vayron, R., et al., Ultrasonic evaluation of dental implant osseointegration. Journal of Biomechanics, 2014. 47(14): p. 3562-3568. https://doi.org/10.1016/j.jbiomech.2014.07.011 [14] Cairns, N.J., et al., Evaluation of modal analysis techniques using physical models to detect osseointegration of implants in transfemoral amputees. Conference proceedings: ... Annual International Conference of the IEEE Engineering in Medicine and Biology Society. IEEE Engineering in Medicine and Biology Society. Conference, 2011: p. 1600-1603. https://doi.org/10.1109/IEMBS.2011.6090463

[15] Dhert, W., et al., A finite element analysis of the push-out test: Influence of test conditions. Journal of Biomedical Materials Research Part A, 1992. 26(1): p. 119-130.

https://doi.org/10.1002/jbm.820260111

[16] Johansson, C.B., L. Sennerby, and T. Albrektsson, A removal torque and histomorphometric study of bone tissue reactions to commercially pure titanium and Vitallium implants. International Journal of Oral \& Maxillofacial Implants, 1991. 6(4).

[17] Chiu, W.K., et al., Vibration-based healing assessment of an internally fixated femur. Journal of Nondestructive Evaluation, Diagnostics and Prognostics of Engineering Systems, 2019. 2(2): p. 021003. https://doi.org/10.1115/1.4043276

[18] Chiu, W.K., et al., Towards a Non-Invasive Technique for Healing Assessment of Internally Fixated Femur. Sensors, 2019. 19(4): p. 857. https://doi.org/10.3390/s19040857

[19] Chiu, W., et al., Effects of mass loading on the viability of assessing the state of healing of a fixated fractured long bone. Journal of Rehabilitation and Assistive Technologies Engineering, 2019. 6: p. 2055668319842806. https://doi.org/10.1177/2055668319842806

[20] Li, P., N. Jones, and P. Gregg, Vibration analysis in the detection of total hip prosthetic loosening. Medical engineering \& physics, 1996. 18(7): p. 596-600. https://doi.org/10.1016/1350-4533(96)00004-5

[21] Varini, E., et al., Assessment of implant stability of cementless hip prostheses through the frequency response function of the stem-bone system. Sensors and Actuators A: Physical, 2010. 163(2): p. 526-532. https://doi.org/10.1016/j.sna.2010.08.029

[22] Jaecques, S.V., C. Pastrav, and G. Van der Perre. Analysis of the fixation quality of total hip replacements using a vibrational technique. in ASME 7th Biennial Conference on Engineering Systems Design and Analysis. 2004. American Society of Mechanical Engineers. https://doi.org/10.1115/ESDA2004-58581

[23] Alshuhri, A.A., et al., Development of a non-invasive diagnostic technique for acetabular component loosening in total hip replacements. Med Eng Phys, 2015. 37(8): p. 739-45.

https://doi.org/10.1016/j.medengphy.2015.05.012

[24] Bediz, B., H.N. Özgüven, and F. Korkusuz, Vibration measurements predict the mechanical properties of human tibia. Clinical biomechanics, 2010. 25(4): p. 365-371.

https://doi.org/10.1016/j.clinbiomech.2010.01.002

[25] Jaecques, S., et al., Vibration analysis of orthopaedic implant stability: exploratory finite element modelling. Proceedings of the Belgian Day on Biomedical engineering, 2002. 
[26] Huang, H.M., et al., Early detection of implant healing process using resonance frequency analysis. Clinical oral implants research, 2003. 14(4): p. 437-443. https://doi.org/10.1034/j.16000501.2003.00818.x

[27] Cairns, N.J., et al., Ability of modal analysis to detect osseointegration of implants in transfemoral amputees: a physical model study. Med Biol Eng Comput, 2013. 51(1-2): p. 39-47. https://doi.org/10.1007/s11517-012-0962-0

[28] Qi, G., W.P. Mouchon, and T.E. Tan, How much can a vibrational diagnostic tool reveal in total hip arthroplasty loosening? Clinical Biomechanics, 2003. 18(5): p. 444-458. https://doi.org/10.1016/S0268-0033(03)00051-2

[29] Rieger, J.S., et al., Loosening detection of the femoral component of hip prostheses with extracorporeal shockwaves: a pilot study. Med Eng Phys, 2015. 37(2): p. 157-64. https://doi.org/10.1016/j.medengphy.2014.11.011

[30] Dong, Y., et al., Finite Element Analysis of Absorbable Sheath to Prevent Stress Shielding of Tibial Interlocking Intramedullary Nail. IOP Conference Series: Materials Science and Engineering, 2017. 224. https://doi.org/10.1088/1757-899X/224/1/012052

[31] Helgason, B., et al., Risk of failure during gait for direct skeletal attachment of a femoral prosthesis: a finite element study. Medical engineering \& physics, 2009. 31(5): p. 595-600. https://doi.org/10.1016/j.medengphy.2008.11.015

[32] Pal, S., Design of artificial human joints \& organs. 2014: Springer. https://doi.org/10.1007/978-1-4614-6255-2

[33] Oftadeh, R., et al., Biomechanics and mechanobiology of trabecular bone: a review. Journal of biomechanical engineering, 2015. 137(1). https://doi.org/10.1115/1.4029176

[34] Dalstra, M., et al., Mechanical and textural properties of pelvic trabecular bone. Journal of biomechanics, 1993. 26(4-5): p. 523-535. https://doi.org/10.1016/0021-9290(93)90014-6

[35] RUSS, M., et al., Development of a Novel Osseointegrated Endoprosthesis, Combing Orthopaedic and Engineering Design Principles, and Structural Health Monitoring Conc. Structural Health Monitoring 2017, 2017(shm). https://doi.org/10.12783/shm2017/14244

[36] Wang, W. and J.P. Lynch, IWSHM 2017: Application of guided wave methods to quantitatively assess healing in osseointegrated prostheses. Structural Health Monitoring, 2018. 17(6): p. 1377-1392. https://doi.org/10.1177/1475921718782399

[37] Vien, B.S., et al., A Quantitative Approach for the Bone-implant Osseointegration Assessment Based on Ultrasonic Elastic Guided Waves. Sensors, 2019. 19(3). https://doi.org/10.3390/s19030454

[38] Chiu, W.K., et al., Healing assessment of fractured femur treated with an intramedullary nail. Structural Health Monitoring, 2019: p. 1475921718816781. https://doi.org/10.1177/1475921718816781

[39] Claes, L.E. and J.L. Cunningham, Monitoring the mechanical properties of healing bone. Clin Orthop Relat Res, 2009. 467(8): p. 1964-71. https://doi.org/10.1007/s11999-009-0752-7 